\title{
Cyclosporin-A therapy of chronic lymphoblastic leukemia related pancytopenia: a case report
}

ying Wang ${ }^{1}$, Yan $\mathrm{Gao}^{2}$, Junxia Huang ${ }^{3}$, Tianlan $\mathrm{Li}^{3}$, Chunxia $\mathrm{Mao}^{3}$, Shanshan $\mathrm{Liu}^{3}$, Yujie $\mathrm{XU}^{3}$, Han $\mathrm{Xu}^{3}$, and Xianqi Feng ${ }^{3}$

${ }^{1}$ Qingdao University

${ }^{2}$ Affiliated Hospital of Medical College Qingdao University

${ }^{3}$ The Affiliated Hospital of Qingdao University

October 30, 2021

\begin{abstract}
Here we present a case report of a chronic lymphoblastic leukemia-related pancytopenia patient where after treatment with Cyclosporin-A, chronic lymphoblastic leukemia has effectively remitted. To further understand the effect of Cyclosporin-A in immune micro-environment reconstruction and its anti-leukemia potential.
\end{abstract}

Cyclosporin-A therapy of chronic lymphoblastic leukemia relatedpancytopenia: a case report Mengying Wang $\mathrm{MS}^{1,2}$, Ruoyu Meng $\mathrm{PhD}^{3}$, Yan Gao $\mathrm{PhD}^{2}$, Junxia Huang $\mathrm{PhD}^{2}$, Tianlan $\mathrm{Li} \mathrm{PhD}^{2}$, Chunxia Mao $\mathrm{MS}^{2}$, Shanshan Liu $\mathrm{PhD}^{2}$, Yujie $\mathrm{Xu} \mathrm{PhD}^{2}$, Han $\mathrm{Xu} \mathrm{MS}^{1,2}$, Xianqi Feng $\mathrm{PhD}^{2, *}$

${ }^{1}$ Faculty of Medicine, Qingdao University, Department of Hematology, the Affiliated Hospital of Qingdao University, Qingdao 266000, China

${ }^{2}$ Department of Hematology, the Affiliated Hospital of Qingdao University, Qingdao 266000 China

${ }^{3}$ Department of Physiology, Chonbuk National University Medical School, Jeonju 54907, Korea

*Correspondence

Xianqi Feng Ph.D., Department of Hematology, the Affiliated Hospital of Qingdao University, No. 1677, Mount Wutai Street, Huangdao District, Qingdao Shandong Province, China

E-mail address: qdfxq2005@163.com

Tel: 86-17853299071

\section{Data availability statement}

The data that support the findings of this study are available from the corresponding author upon reasonable request.

\section{Funding information}

This paper is not funded

\section{Conflict of Interest}

The authors declare no conflict of interest.

statement of author contribution 
Mengying Wang conceived and analyzed the case report. Ruoyu Meng drafted the manuscript. Xianqi Feng drafted and revised the manuscript. Yan Gao, Junxia Huang, Tianlan Li, Chunxia Mao, Shanshan Liu, Yujie Xu, and Han Xu MS collected data and analyzed the case report.

\section{Patient consent statement}

The patient discussed in this case report gave informed consent and agreed to participate in the study.

\section{ABSTRACT}

What is known and objective : To explore the clinical characteristics, diagnosis, and treatment of chronic lymphocytic leukemia (CLL) with secondary pancytopenia.

Case summary : Here, a case of pancytopenia secondary to CLL is reported. Additionally, a review of relevant CLL literature was conducted to summarize its diagnosis, clinical characteristics, treatment history, and experience. After treatment with cyclosporin A (CsA), the patient's CLL continued to resolve and hematopoiesis returned to normal.

What is new and Conclusion : CsA therapy resulted in improved patient outcomes. However, the mechanism by which CSA rebuilds the immune microenvironment and its anti-leukemia effect in the body remains to be studied.

Keyword s: case report, chronic lymphoblastic leukemia, Cyclosporin-A, immune micro-environment

\section{What is known and objective}

Chronic lymphoblastic leukemia (CLL) is a chronic B-cell proliferative disorder. The median age of patients suffering from CLL is 67-72 years and the incidence rate is higher in males than in females $(2: 1) .{ }^{1}$ Although autoimmune cytopenia (AIC) frequently co-occurs with chronic CLL, its relationship to pancytopenia is rarely reported and its pathogenesis remains unknown. Recently, the treatment of CLL-related pancytopenia using Cyclosporin-A (CsA) induced a prolonged remission of CLL. We hypothesize that CsA may have the dual effect of acting as an immunosuppressant whilst simultaneously being able to induce immune microenvironment reconstruction which, together, may facilitate its anti-leukemia potential; however, this need to be verified. In the current case study, a CLL patient presented with secondary pancytopenia and did not respond adequately to treatment using stimulating factors and was reliant on blood transfusion. After CsA treatment, routine blood indices rose to the normal range indicating that CLL has been remitted.

\section{Case summary}

In March 2016, a 63-year-old female patient was admitted to The Affiliated Hospital of Qingdao University due to the enlargement of the bilateral cervical lymph nodes. The patient had no obvious medical history. Upon systemic examination, several enlarged lymph nodes were detected in both the cervical and inguinal locations. The volume of the largest cervical lymph node was approximately $3 \times 2 \mathrm{~cm}$; however, no tenderness or adhesion was detected. Further, the spleen was enlarged with Line I, Line II, and Line III measuring $6 \mathrm{~cm}$, $7 \mathrm{~cm}$, and $2 \mathrm{~cm}$, respectively. No tenderness was noted. Hematological examination revealed the following results: WBC $87.40 \times 10^{9} / \mathrm{L}$, NEU $4.95 \times 10^{9} / \mathrm{L}, \mathrm{RBC} 3.43 \times 10^{12} / \mathrm{L}, \mathrm{HGB} 94 \mathrm{~g} / \mathrm{L}, \mathrm{PLT} 86 \times 10^{9} / \mathrm{L}, \mathrm{LYM}$ $81.03 \times 10^{9} / \mathrm{L} ;$

An examination of the bone marrow revealed that the proliferation of granulocytes was inhibited. The proliferation of erythrocytes was normal. Further, erythroblasts of slightly different sizes were noted. Lymphatic hyperplasia was significant (88.5\%) with most cells being abnormal, small lymphocytes. Morphologically, the lymphocytes were small, round, possessed large round nuclei, dense chromatin, and little sky-blue cytoplasm. Together, these observations are indicative of lymphoproliferative disease (LDP). Mature lymphocytes and abnormal cell populations expressing CD19, CD5, CD43, CD23, CD200, and weakly expressing of CD20, CD79b, CD38, CD81, CD22, Kappa, and SIgD accounted for $93.2 \%$ and $91.78 \%$ of the nuclear cells, respectively. Together, these lines of evidence are indicative of a CLL/SLL phenotype. Fluorescence in situ hybridization (FISH) analysis highlighted that the patients' samples was positive for the rearrangement of 
IGH and IGK. Further, the patient was negative for the ATM, CCND1/IGH, CEP12, P53, and RB-1 genes. Based on these data, the patient was diagnosed with CLL (Rai Stage IV; Binet Stage C; High-risk group).

From the $28^{\text {th }}$ of March 2016, a 5-course FC chemotherapy regimen (on days 1-3, fludarabine $50 \mathrm{mg}$ and cyclophosphamide $0.4 \mathrm{~g}$ ) was initiated following symptomatic supportive treatment. Bone marrow suppression was observed after each course of chemotherapy, hematopoiesis returned to normal after symptomatic supportive treatment, e.g., stimulating the hematopoiesis. From the examination of the bone marrow, the patient was determined to be in complete remission (CR). On the $22^{\text {nd }}$ of September 2016, the sixth course of FC chemotherapy was started, and the patient was discharged after the bone marrow hematopoiesis was recovered. No additional chemotherapy was carried out.

The patient was admitted to the hospital again on the $3^{\text {rd }}$ of November 2016. Hematological examination revealed: WBC $\left(2.18 \times 10^{9} / \mathrm{L}\right), \mathrm{NEU}\left(1.41 \times 10^{9} / \mathrm{L}\right), \operatorname{RBC}\left(2.33 \times 10^{12} / \mathrm{L}\right), \mathrm{HGB}(64 \mathrm{~g} / \mathrm{L})$, PLT $\left(15 \times 10^{9} / \mathrm{L}\right)$, and LYM $\left(1.29 \times 10^{9} / \mathrm{L}\right)$. Further, the absolute number of the reticulocytes was decreased. Additionally, there was no evidence of viral infection or hemolysis. Symptomatic supportive treatment to stimulate hematopoiesis was given; however, these treatments were not effective and additional blood transfusions were necessary. On the $15^{\text {th }}$ of November 2016, the re-examination of bone marrow morphology found that the proliferation of bone marrow cells was less active. Cells at each stage were rare and the size and morphology of the erythroblasts were good. Furthermore, $18 \%$ were lymphocytes and an occasional $1 \%$ were prolymphocytes. On the whole film, no megakaryocytes were observed and platelets were rare which is indicative of a poor proliferation of bone marrow cells. No clonal abnormality on the chromosome was observed. The patient refused a multi-site bone marrow biopsy for personal reasons. On the $24^{\text {th }}$ of November 2016, after relevant disease progression was excluded, additional CsA (100 mg) was supplemented empirically, twice a day. At the same time, hematopoiesis stimulation and intermittent transfusion of blood components were given. The patient's platelet level gradually increased, and the hemoglobin count also increased to $70 \mathrm{~g} / \mathrm{L}$ where it stabilized. The patient was successfully weaned off the blood transfusions. The patient did not receive FC chemotherapy, however, oral CsA treatment was continued at home. On the $12^{\text {th }}$ of January 2018, a follow-up bone marrow biopsy revealed that no clonal abnormalities were observed in the B-lymphocytes and that there was a low proliferation of bone marrow cells ( $\sim 30 \%)$. FISH analysis was negative. To date, the patient has remained in remission of CLL 5 years and 3 months as confirmed by routine assessments. 


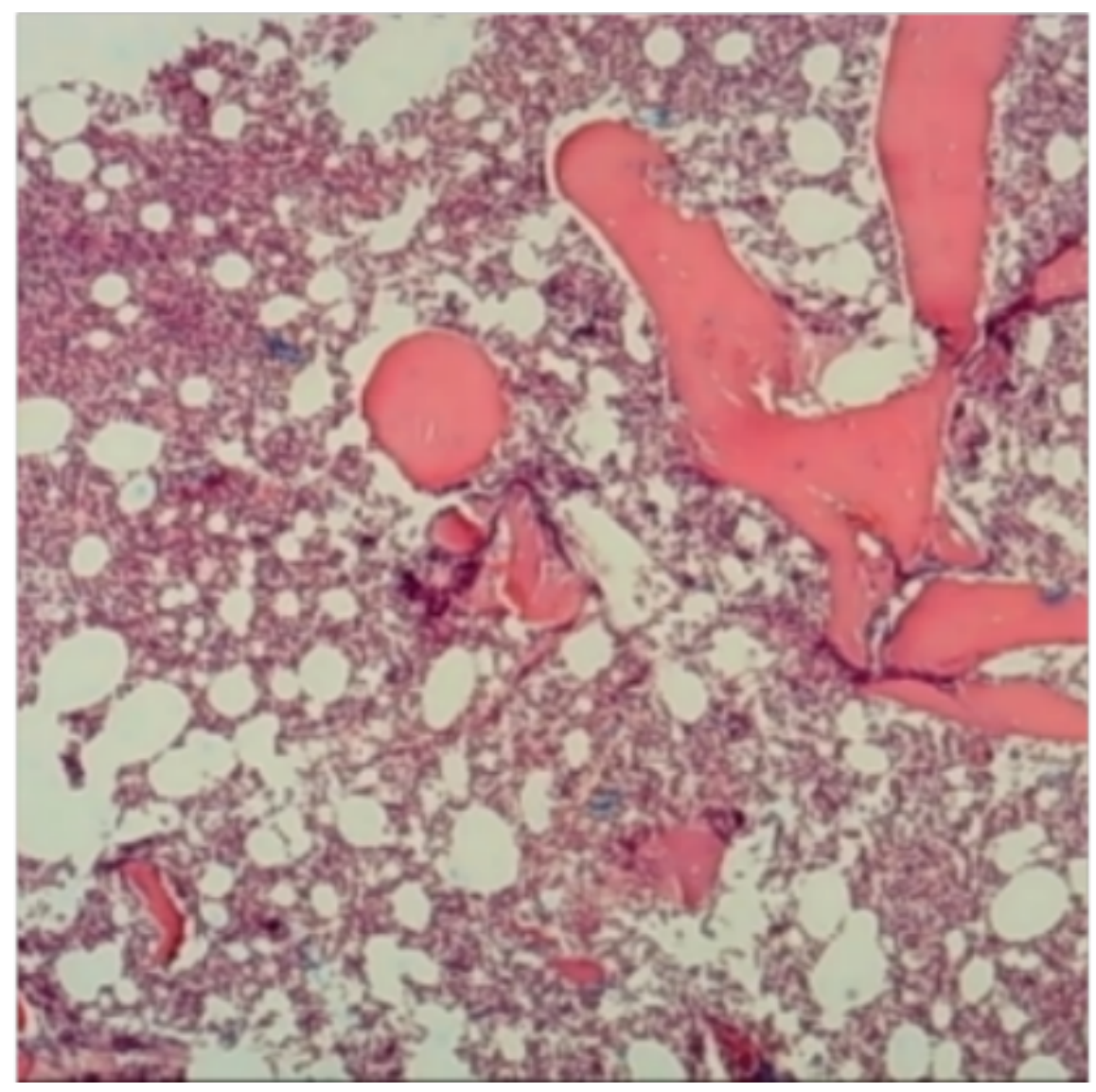




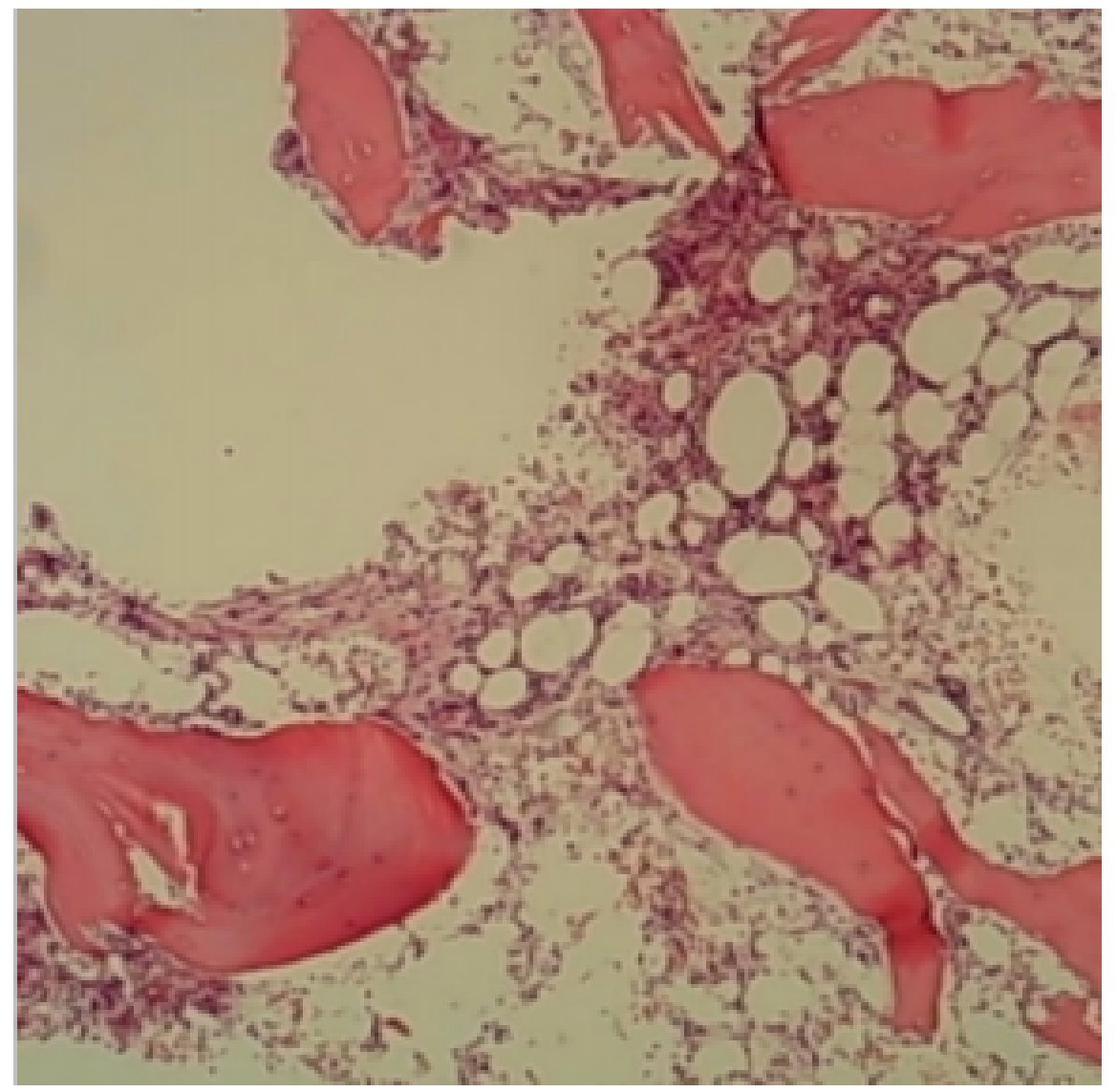




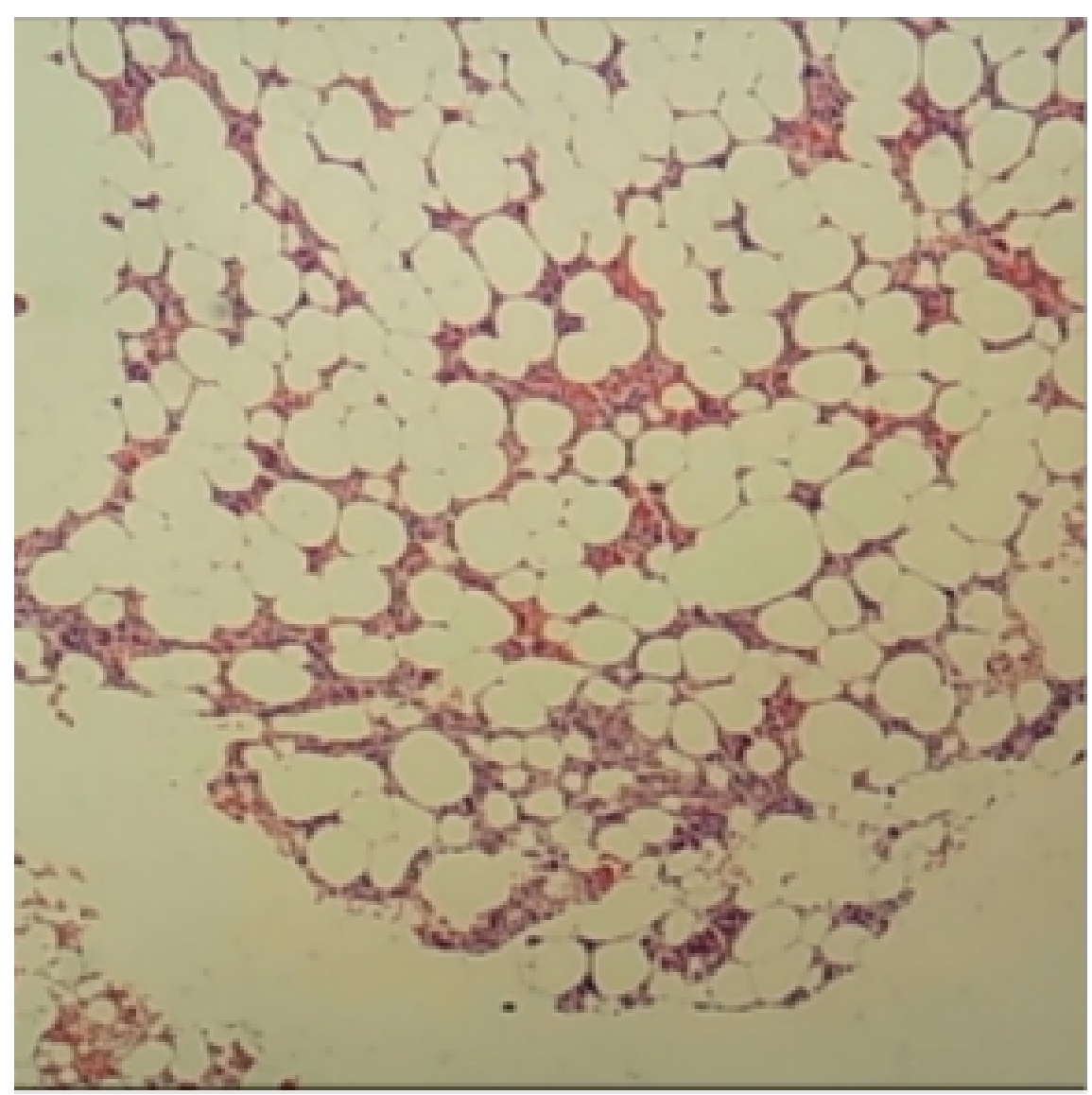




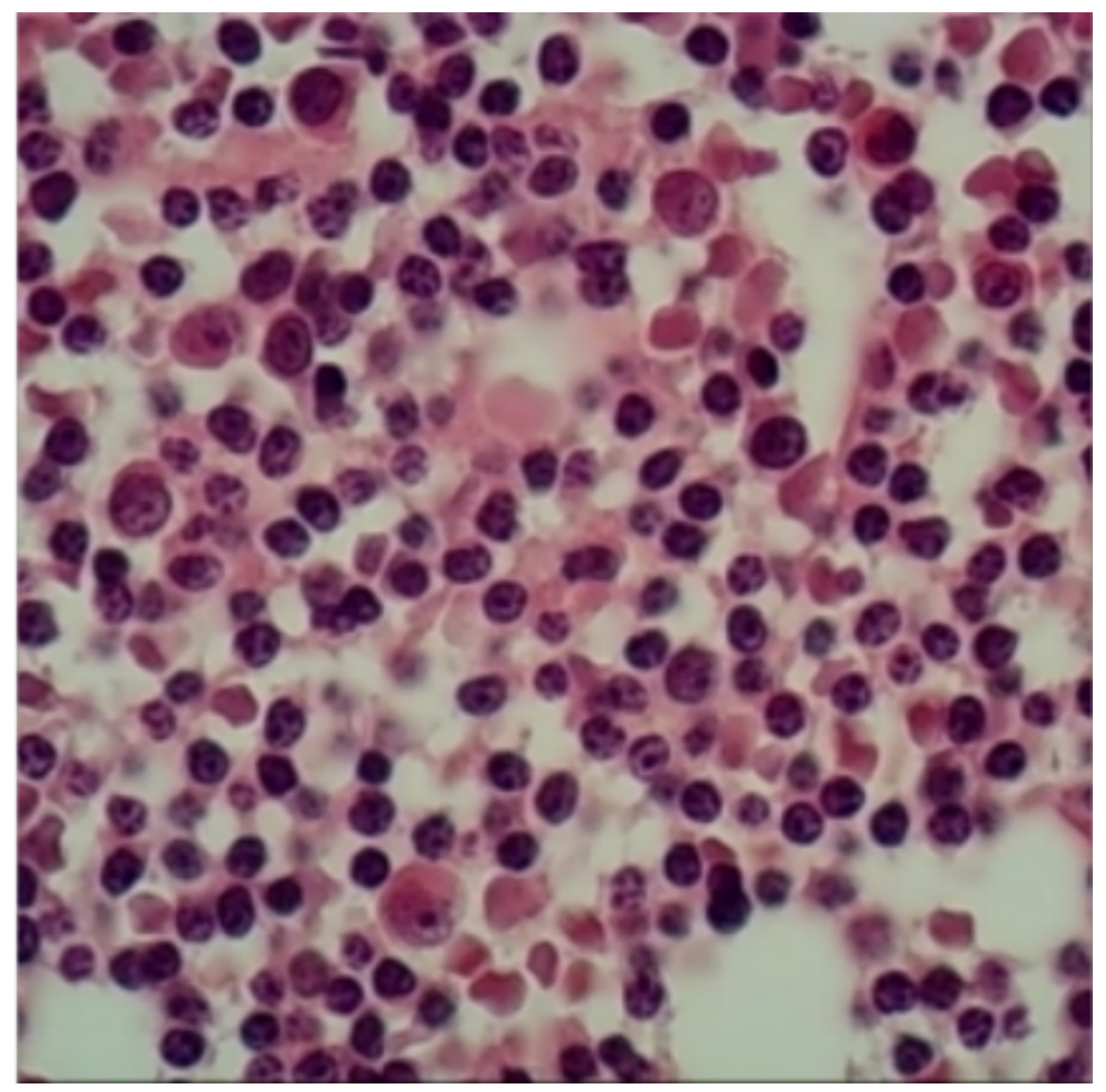




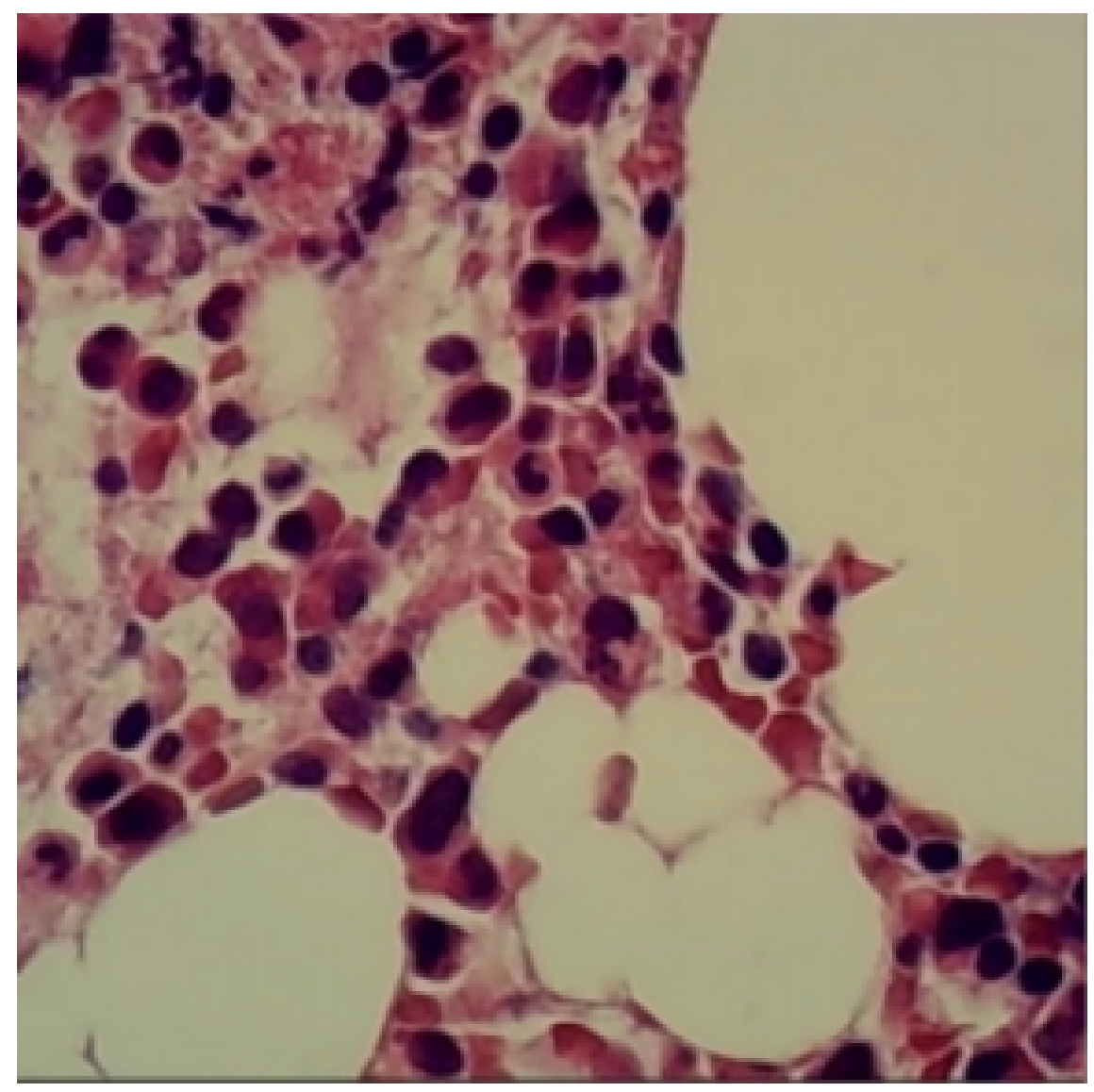




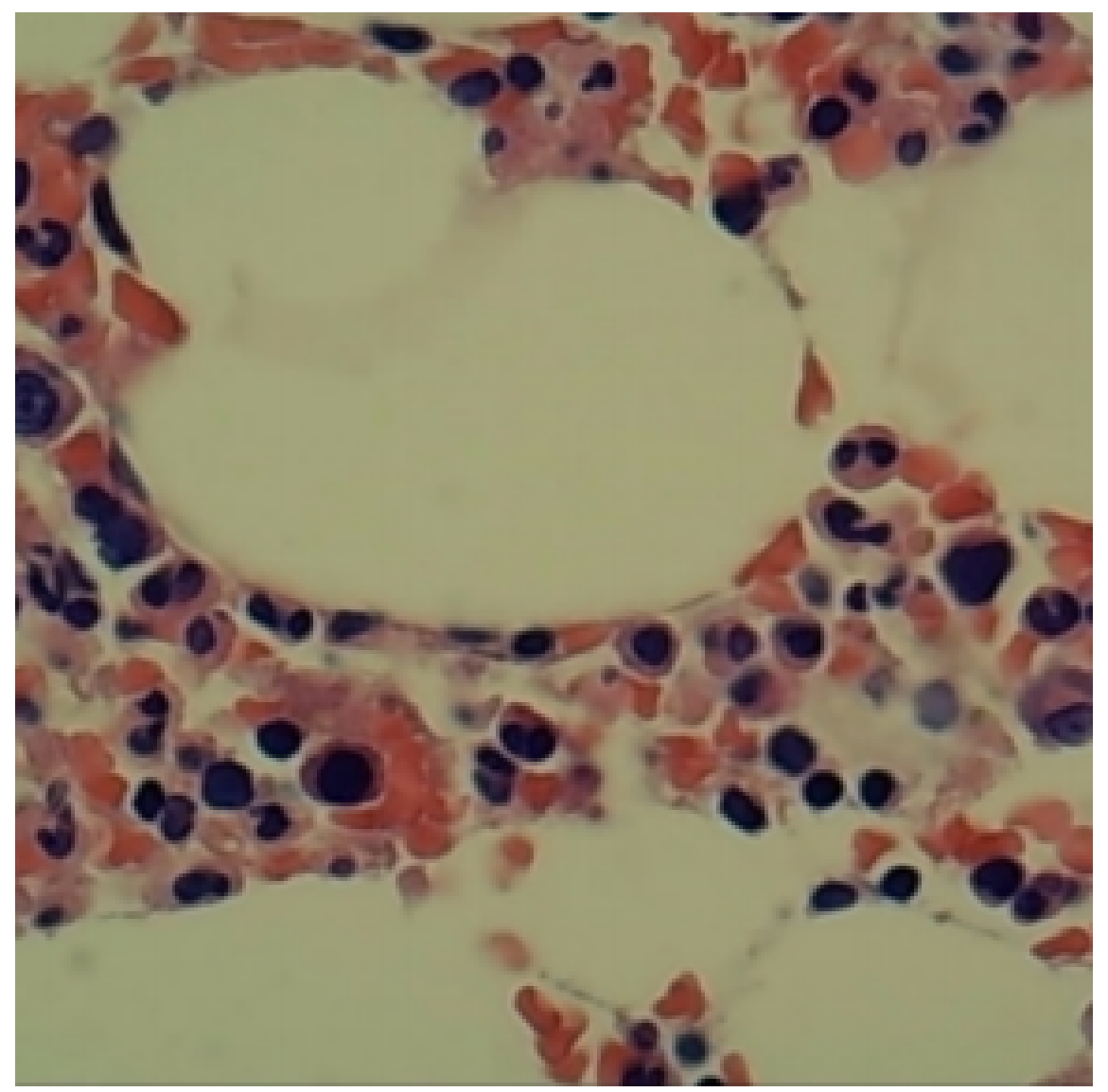

Figure 1 (March 16, 2016) Bone marrow pathology at initial diagnosis: hyperactive myelodysplasia (90\%), lymphocytosis; (2016-11-15) Bone marrow pathology: poor myelodysplasia, rare megakaryocytes; Bone marrow pathology after treatment showed low myelodysplasia (about 30\%) and rare megakaryocytes.

\section{What is new and Conclusion}

The manifestations of CLL are excessive proliferation and aggregation of clonal B lymphocytes in peripheral blood, bone marrow, lymphaden, and spleen. Clonal B lymphocytes are similar to mature lymphocytes in morphology. However, these cells possess abnormal immune expression and dysregulated cell functioning. Furthermore, despite having the typical immunophenotypes of clonal B lymphocytes (CD5 ${ }^{+}, \mathrm{CD}_{10}{ }^{-}, \mathrm{CD} 19^{+}$, $\mathrm{FMC}^{-}, \mathrm{CD}_{2} 3^{+}, \mathrm{CD} 43^{+/-}$and $\mathrm{CCND1}^{-}$), the cells are considered abnormal. In the 1980s, the ability to assess genomic sequences for markers of disease facilitated a significant breakthrough in CCL diagnosis and therapy. Combination treatment using fludarabine and cyclophosphamide (FC) has become the basic recommendation which is stated in the NCCN guidelines. ${ }^{2,3}$ But CLL remains an incurable hematologic malignant disease.

CLL can cause various complications, especially infection and autoimmune diseases. Globally, the incidence of autoimmune diseases is approximately $10-25 \%$ and often affects the blood system. The most common autoimmune diseases are autoimmune cytopenias (AIC), developed in 4-7\% of CLL patients. Among these cases, pure erythrocyte aplastic anemia (PRCA) is a rare disorder that is often seen in the terminal stage of disease in fludarabine-treated patients. ${ }^{4}{ }^{5} \mathrm{PRCA}$ may be related to immune dysfunction induced by immune inhibitors, but this remains to be elucidated. Immune-mediated hypocytosis occurs frequently in CLL, but only two cases have reported CLL with secondary pancytopenia. ${ }^{6,7}$ The pathogenesis of CLL-related AIC is associated with $\mathrm{T}$ lymphocyte dysfunction, clonally proliferating B lymphocyte antigen presentation, and autoantibody production by normal residual B lymphocytes. ${ }^{8,9}$ Previous studies have shown that these 
antibodies directly inhibited erythropoiesis and thrombocytopoiesis. ${ }^{10}, 11$ Additionally, CLL-related AIC can easily be confused with CLL progression, chemotherapy-related marrow suppression, myelodysplastic syndrome, and acute arrest of hemopoiesis. Immunohistochemical analyses and the assessment of the recovery period after treatment provide key strategies to differentiate and diagnose these diseases.

Lad et $\mathrm{al}^{12}$ reported that dysfunctional $\mathrm{T}$ lymphocytes in CLL, especially at the late stage, may explain the pathogenesis of CLL-associated AIC. Therefore, although no direct evidence has shown that CLL patients have a significant tendency to develop auto-immune disorders, the correlation between CLL and non-hematological autoimmunity should not be ignored. In the current case report, the increased frequency of inhibitory T lymphocytes was indicative of immunologic derangement in the patient. Further, these data suggest that the induction of autoantibodies and $\mathrm{T}$ lymphocyte dysfunction may play important roles in our case. CsA, an immunomodulator, can regulate $\mathrm{T}$ lymphocyte function and suppress the release of lymphokines such as IL-2, IL-4, TNF, and IFN from antigen-activated T lymphocytes. We further explored how CsA regulates immunologic derangement in CLL patients and whether CsA can facilitate complete remission. Previously, a study of 31 cases where CsA treatment was used to treat CLL-related AIC confirmed its effectiveness. In that study, 19 of $29(66 \%)$ thrombocytopenic patients and 11 of 16 (69\%) anemic patients were remitted following treatment. Additionally, a reduction of tumor load and lymph nodes was observed in some patients. ${ }^{13}$ Zhao et al ${ }^{14}$ suggested that CsA can regulate the immune micro-environment, recover $\mathrm{T}$ lymphocyte function, inhibit auto-antibodies, eliminate tumor cells, and reduce tumor cell escape. These mechanisms may be the methods by which CsA sustained remitted CLL and exerted synergistic effects in leukemia therapy. However, the underlying mechanism of CsA in CLL cell lines remains unknown. Furthermore, any evidence for the treatment of CLL-related autoimmune disease using CsA treatment is mostly found in cases reports. Together, data in the literature highlight the urgent need for the assessment of CsA in a large-scale, sufficiently powered experimental setting. Additionally, after the reduction of tumor load in CLL, the ability of CsA to regulate marrow immune-environment and exert synergistic anti-tumor effects need to be further explored.

In conclusion, we reported a CLL patient with related pancytopenia who showed an obvious and complete remission of the disease after CsA treatment. These findings provide new insights into CLL treatment and help to inform future studies.

\section{REFERENCES}

1. Baldoni S, Del Papa B, De Falco F, et al. Notch1 activation negatively impacts on chronic lymphocytic leukemia outcome and is not correlated to the notch1 and ighv mutational status. Front Oncol 2021;11:668573. 2. Woyach JA. What is the optimal management of older cll patients? Best Pract Res Clin Haematol 2018;31(1):83-89. 3. Woyach JA. Treatment-naive cll: Lessons from phase 2 and phase 3 clinical trials. Blood 2019;134(21):1796-1801. 4. Quinquenel A, Aurran-Schleinitz T, Clavert A, et al. Diagnosis and treatment of chronic lymphocytic leukemia: Recommendations of the french cll study group (filo). Hemasphere 2020;4(5):e473. 5. Vitale C, Montalbano MC, Salvetti C, et al. Autoimmune complications in chronic lymphocytic leukemia in the era of targeted drugs. Cancers (Basel) 2020;12(282). 10.3390/cancers12020282. 6. Singal R, Winfield DA, Greaves M. Bone marrow aplasia in b cell chronic lymphocytic leukaemia: Successful treatment with antithymocyte globulin. J Clin Pathol 1991;44(11):954-956. 7. Zonder JA, Keating M, Schiffer CA. Chronic lymphocytic leukemia presenting in association with aplastic anemia. Am J Hematol 2002;71(4):323-327. 8. Hochman MJ, Martin DB. Coincidence of autoimmune hemolytic anemia and pure red cell aplasia in a patient with cll. Ann Hematol 2021;100(2):579-580. 9. Reinart N, Nguyen $\mathrm{PH}$, Boucas J, et al. Delayed development of chronic lymphocytic leukemia in the absence of macrophage migration inhibitory factor. Blood 2013;121(5):812-821. 10. Wherry EJ. T cell exhaustion. Nat Immunol 2011;12(6):492-499. 11. Forconi F, Moss P. Perturbation of the normal immune system in patients with cll. Blood 2015;126(5):573-581. 12. Lad D, Hoeppli R, Huang Q, et al. Regulatory t-cells drive immune dysfunction in cll. Leuk Lymphoma 2018;59(2):486-489. 13. Wright SJ, Keating MJ. Cyclosporine a in chronic lymphocytic leukemia: Dual anti-leukemic and immunosuppressive role? Leuk Lymphoma 1995;20(1-2):131136. 14. Zhao X, Zhou K, Jing L, et al. Treatment of t-cell large granular lymphocyte leukemia with 
cyclosporine a: Experience in a chinese single institution. Leuk Res 2013;37(5):547-551. 